\title{
Bone Protective Treatment: Prevention of Cancer Treatment Associated Bone Loss and Bone Metastases
}

\author{
Ömer DizDAR
}

Department of Medical Oncology, Hacettepe University Cancer Institute, Ankara-Turkey

\section{Prevention of Bone Loss Associated with Cancer Treatment}

In cancer patients, both chronic local or systemic inflammation caused by cancer itself and anti-cancer treatment, particularly endocrine therapy, can lead to bone problems. Chronic inflammation affects osteoblast and osteoclast activity through inflammatory mediators, increases bone resorption, and reduces new bone formation. Androgens and estrogen have a very important role in maintaining bone mass. Cancer treatment-associated loss of bone mineral density is an important problem, particularly in people receiving endocrine therapy for breast or prostate cancer. This is due to the fact that endocrine therapies aimed at reducing androgen and estrogen levels accelerate bone destruction. For example, the lumbar spinal bone mineral density (BMD) decrease rate is $1 \%$ per year in the early stages of menopause; however, this rate increases to $7 \%$ per year when aromatase inhibitor and GnRH analog are used together. Annual loss of BMD in chemotherapy-induced ovarian failure has been reported to be 7.7\%.[1] In patients undergoing aromatase inhibitor therapy without bone-sparing treatment, the risk of fracture may increase up to $20 \%$ in the $5^{\text {th }}$ year.[2,3] The risk of osteoporosis reaches $80 \%$ in prostate cancer patients who have been receiving androgen deprivation therapy (ADT) for ten years or more.[4]

\section{Risk Factors}

Risk factors for osteoporotic fractures are: advanced age (women $>65$, men $>70$ ), early menopause, smoking and alcohol consumption, history of non-traumatic fracture, hypogonadism, inactivity, steroid use for more than 3-6 months, low body weight, menopause, and family history of hip fracture.[5] In addition to these factors, malnutrition, radiotherapy, sarcopenia and related inactivity, and fatigue can also increase the risk of osteoporosis in cancer patients. Standard risk assessment tools, such as "fracture risk assessment tool" (FRAX) recommended by the World Health Organization (WHO), can be used to determine risk. FRAX is used to estimate the 10 -year probability of an osteoporotic fracture by taking into account the general characteristics of patients and risk factors for osteoporosis. Patients with a 10-year hip fracture risk of $3 \%$ or more, or an overall fracture risk of $20 \%$ or more, are considered to be at high risk and require intervention. The use of anti-estrogen or anti-androgen therapy and the use of corticosteroids for 3 months or longer should be considered in risk assessment. Although data on the use of tools such as FRAX in cancer patients are limited, they are still clinically useful.

\section{Follow-up and Treatment}

Cancer patients with the above-mentioned risk factors for osteoporosis should be followed up for bone fracture risk and BMD should be evaluated periodically with dual-energy $\mathrm{x}$-ray absorbtiometry (DXA). DXA is ideally performed every 2 years; however, it can be performed more frequently, depending on risk factors and baseline measurement results, but the interval should not be shorter than one year.[6]

To prevent treatment-related bone loss, it is very important to exercise and take adequate amounts of calcium (1200 mg) and vitamin D (800-1000 IU) regularly, and if the daily consumption is low, additional calcium and vitamin D support should be provided.

\section{Dr. Ömer DiZDAR}

Hacettepe Üniversitesi Kanser Enstitüsü,

Tıbbi Onkoloji Anabilim Dalı,

Ankara-Turkey

E-mail: omer.dizdar@hacettepe.edu.tr 
The exercise program should be designed according to the patient's condition and capacity and may include endurance, resistance, and weight-bearing exercises. Smoking and alcohol consumption should be avoided. In addition to these, the use of anti-resorptive therapy is essential in a majority of patients. As per the ESMO guidelines, treatment with bisphosphonate or denosumab should be initiated in patients receiving endocrine treatment for cancer and who have a BMD T score of less than -2 . In patients with a BMD $\mathrm{T}$ score greater than -2 , treatment decision should be made according to risk factors (age $>65$, $\mathrm{T}$ score $<-1.5$, smoking, BMI $<24$, family history of hip fracture, personal history of fragility fracture above age 50 , and oral glucocorticoid use for $>6$ months). In patients exposed to 2 or more of the aforementioned risk factors, anti-resorptive therapy should be commenced.

Both oral or intravenous bisphosphonates and denosumab are active treatment options to increase BMD. In prospective randomized trials carried out in cancer patients, these drugs have been shown to prevent bone loss, thus reducing fracture risk. The doses used in these studies are generally similar to the dose in the treatment of senile osteoporosis. In a study comparing the efficacy of $35 \mathrm{mg}$ risedronate administered once in a week versus placebo in patients with breast cancer, it was shown that risedronate prevented bone loss and reduced bone turnover.[7] In the SABER study, the use of risedronate together with calcium and vitamin D supplements in patients using aromatase inhibitors provided a significant increase in hip and vertebral BMD. [8] Similarly, $150 \mathrm{mg}$ ibandronate administered once a month in breast cancer patients using aromatase inhibitor was revealed to improve BMD and reduction of bone loss as compared to placebo.[9] In the ABCSG-12 study, it was shown that $4 \mathrm{mg}$ of zoledronate administered every 6 months prevented bone loss in women with premenopausal breast cancer who use OFS with tamoxifen or aromatase inhibitor.[10] Z_FAST, ZOFAST, and E-ZO-FAST studies compared the concurrent use of zoledronate with an aromatase inhibitor in post-menopausal women (early use), and the addition of zoledronate to the treatment when BMD falls below -2 (delayed use). In all the 3 studies, early use of zoledronate was found to prevent bone loss.[11-13] It was observed that rapid bone loss in early menopause was prevented with zoledronate treatment, and an increase in BMD was achieved with early zoledronate treatment in patients within long-term menopause.[11] These studies were not designed to investigate whether there is a difference in fracture risk between study arms; however, the increase in BMD with early treatment suggests that fracture risk may also be reduced.

In the ABCSG-18 study, it was shown that in postmenopausal breast cancer patients undergoing aromatase inhibitor therapy, the use of denosumab $(60 \mathrm{mg}$ every 6 months) with adequate calcium and vitamin $\mathrm{D}$ supplements reduced the risk of fracture by $50 \%$. This effect was found to be independent of age and baseline BMD.[14] Prior to the initiation of aromatase inhibitor therapy, BMD and other osteoporosis risk factors should be evaluated, and calcium, phosphorus, creatinine, and vitamin $\mathrm{D}$ levels should be assessed.

In 1468 patients with hormone sensitive prostate cancer who did not have metastasis and received $\mathrm{ADT}$, the use of denosumab at a dose of $60 \mathrm{mg}$ every 6 months resulted in an improvement in BMD and a $62 \%$ reduction in the risk of vertebral fracture after 3 years $(1.5 \%$ in the denosumab arm versus $3.9 \%$ in the placebo arm).[15]

In conclusion, in patients at high risk for osteoporosis, use of oral treatment options such as alendronate, risedronate, and ibandronate, or parenteral treatment with denosumab $60 \mathrm{mg}$ every 6 months and zoledronate $4 \mathrm{mg}$ every 6 months was proved to inhibit bone loss and osteoporosis in cancer patients, and their use is recommended in current guidelines. Adherence to treatment is important in these patients, since a significant proportion of patients can discontinue oral or parenteral treatments at the end of 1 year. Osteonecrosis of the jaw in osteoporosis treatment doses is extremely rare. Nevertheless, dental examination should be done before treatment, and attention should be paid to oral hygiene and invasive dental procedures should be avoided during treatment.[6]

\section{Prevention of Bone Metastases}

It has been known for many years that the microenvironment is as important as the tumor cell itself in tumor pathogenesis and metastasis. According to the "seed and soil" hypothesis, put forward by Stephen Paget in 1889, metastatic tumor cells prefer to metastasize to regions/organs where the local microenvironment is suitable for tumor growth. As per this theory, metastasis can be prevented by modifying the microenvironment where tumor cells might settle. Bisphosphonates act by inhibiting osteoclast activity, thereby inhibiting the secretion of certain mediators such as TGF-beta, which are secreted by osteoclasts and increase the proliferation of tumor cells. There are studies showing that 
bisphosphonates prevent bone metastases in animal models.[16] Bone is the most common site of systemic metastasis in patients with breast and prostate cancer. Studies showing that anti-resorptive drugs can prevent the development of metastasis and prolong survival, especially in patients with breast and prostate cancer, date back 20 years. Most of these studies have shown the benefit of anti-resorptive drugs in patients with low estrogen levels, namely post-menopausal patients or premenopausal patients who use ovarian function suppression (OFS).

In the NSABP-B34 study, the efficacy of adjuvant clodronate use for 3 years among 3323 women with stage I-III breast cancer was investigated. After a median follow-up period of 91 months, there was no difference in disease-free survival (DFS), overall survival (OS), and bone metastasis-free survival. In the subgroup analysis, recurrence-free survival (RFS) and bone metastasis-free survival were in favor of clodronate in women over 50 years of age. A trend in favor of OS with clodronate was observed (HR 0.80, 95\% CI 0.61-1.04; $\mathrm{p}=0.094)$.[17] In other clodronate studies, clinical benefit was observed mostly in postmenopausal women.[18] In the Austrian Breast and Colorectal Cancer Study Group (ABCSG) study, the addition of zoledronate to ovarian suppression therapy every 6 months for 3 years in premenopausal patients with early breast cancer showed DFS benefit $(88.4 \%$ vs. $85 \%$, HR $0.77 ; 95 \%$ CI $0.60-0.99 ; \mathrm{p}=0.042$ ), and OS benefit (96.7\% vs. $94.5 \%$, HR 0.66; 95\% CI 0.43-1.02; Cox $\mathrm{p}=0.064)$, reducing the risk of both local recurrence, distant metastasis, and bone metastasis.[10] In the AZURE study in which the efficacy of zoledronate was investigated in adjuvant therapy, although the DFS and OS benefit was not observed in the whole group, the use of adjuvant zoledronate in the subgroup of patients with menopause for at least 5 years $(n=1041)$ showed the invasive DFS benefit (HR 0.77, 95\% CI 0.63-0.96). Although OS benefit did not reach statistical significance, a positive trend was observed (HR 0.81 (95\% CI 0.63-1.04).[19] These findings were also confirmed in the Early Breast Cancer Trialists' Collaborative Group (EBCTCG) meta-analysis, where data of 18766 patients were analyzed. The study showed that adjuvant bisphosphonate (zoledronate, clodronate, or oral ibandronate) use in patients with natural or $\mathrm{GnRH}$ analog-induced menopause reduced the risk of recurrence (RR 0.86, 95\% CI 0.78-0.94, $2 \mathrm{p}=0.02$ ), risk of bone recurrence (RR0.72, 95\% CI 0.60-0.86, $2 \mathrm{p}=0.0002)$ and the risk of death from breast cancer (RR0.82, 95\% CI 0.73-0.93, $2 \mathrm{p}=0.002$ ). It has been shown that the benefits of bisphosphonates are independent of the sub-type of breast cancer, the type of bisphosphonate used, or the application scheme (for zoledronate).[20] The SWOG S0307 study comparing zoledronate ( $4 \mathrm{mg}$ every 6 months), ibandronate (50 $\mathrm{mg}$ /day), and clodronate $(1600 \mathrm{mg}$ /day) as adjuvant therapy for 3 years in patients with stage I-III breast cancer is the only major phase III study in which the efficacy of various bisphosphonates was compared. In this study, it was shown that the efficacy of all 3 treatments on DFS, OS, and fracture risk was similar. Again, in this study, the frequency of grade 3-4 adverse events was shown to be similar for all the three drugs. [21] Current ESMO and American guidelines recommend the use of zoledronic acid, oral clodronate, or oral ibandronate in the adjuvant treatment of early breast cancer in moderate-to-high risk patients scheduled for adjuvant systemic therapy. Although there is no standard definition of moderate-high risk, patients with an indication for adjuvant systemic treatment or patients with a 10 -year mortality risk of over $12 \%$ due to breast cancer are also considered to be in the appropriate risk profile for bisphosphonate therapy. It is recommended to start bisphosphonate treatment with (neo)adjuvant chemotherapy and continue for 3-5 years for zoledronate, 2-3 years for clodronate, and 3 years for ibandronate.[6] There is no randomized controlled study on the efficacy of alendronate and risedronate, which are commonly used in osteoporosis treatment, as adjuvant therapy to prevent the development of metastasis in breast cancer.

Calcium and vitamin D replacement with bisphosphonate therapy is routinely recommended. It should be confirmed that patients who are scheduled for adjuvant bisphosphonate therapy are patients who meet the definition of menopause, and that FSH, LH, and estradiol levels are consistent with menopause in patients under 60 years of age. Adjuvant bisphosphonate should be recommended in premenopausal patients only in the moderate-high risk group using a $\mathrm{GnRH}$ analog. Bisphosphonate is not recommended for adjuvant therapy in premenopausal patients who do not use a GnRH analog. Normally, patients who have chemotherapy induced amenorrhea should not be accepted as menopausal.[6] Before the use of adjuvant bisphosphonates, patients should be assessed and treated appropriately for dental problems, and patients should be informed about osteonecrosis of the jaw. Patients with symptoms suggesting osteonecrosis of the jaw should be referred to an experienced specialist for treatment. 
There is insufficient data to show that denosumab prevents recurrence in breast cancer. In the ABCSG 18 study, it was shown that denosumab reduced the risk of fracture in patients with post-menopausal early breast cancer compared to placebo, and there was a 3\% difference in DFS in favor of denosumab at 8-year follow-up. However, since the reason for this difference is mostly non-breast second primary cancers and deaths not related to breast cancer, its direct effect on breast cancer is controversial.[14] In the D-CARE study, which is a more recent and larger study, the effectiveness of the use of adjuvant denosumab for 5 years in patients with stage II-III breast cancer was investigated. In this study, it was shown that denosumab did not prevent development of bone metastases in the entire group or the post-menopausal patient subgroup, and did not improve DFS and OS.[22] These findings suggest that the effects of denosumab and bisphosphonates on metastasis biology may be different. Therefore, denosumab is not recommended to be used as adjuvant therapy to prevent the development of metastasis.

There are no studies showing that bisphosphonates have a positive effect on preventing disease recurrence or improving DFS/OS in solid tumors other than breast cancer, and their use for this purpose is not recommended. Although denosumab prolongs the time to bone metastases in patients with prostate cancer, its contribution to survival has not been confirmed and its use is not recommended for this purpose due to $5 \%$ risk of osteonecrosis of the jaw.[23]

\section{References}

1. Shapiro CL, Van Poznak C, Lacchetti C, Kirshner J, Eastell R, Gagel R, et al. Management of osteoporosis in survivors of adult cancers with nonmetastatic disease: ASCO Clinical Practice Guideline. J Clin Oncol 2019;37(31):2916-46.

2. Edwards BJ, Gradishar WJ, Smith ME, Pacheco JA, Holbrook J, McKoy JM, et al. Elevated incidence of fractures in women with invasive breast cancer. Osteoporos Int 2016;27(2):499-507.

3. Melton LJ $3^{\text {rd }}$, Hartmann LC, Achenbach SJ, Atkinson EJ, Therneau TM, Khosla S. Fracture risk in women with breast cancer: a population-based study. J Bone Miner Res 2012;27(5):1196-205.

4. Morote J, Morin JP, Orsola A, Abascal JM, Salvador C, Trilla E, et al. Prevalence of osteoporosis during longterm androgen deprivation therapy in patients with prostate cancer. Urology 2007;69(3):500-4.

5. Viswanathan M, Reddy S, Berkman N, Cullen K, Mid- dleton JC, Nicholson WK, et al. Screening to prevent osteoporotic fractures: An evidence review for the U.S. Preventive Services Task Force [Internet]. Rockville (MD): Agency for Healthcare Research and Quality (US); 2018 Jun. Report No.: 15-05226-EF-1.

6. Coleman R, Hadji P, Body JJ, Santini D, Chow E, Terpos E, et al. Bone health in cancer: ESMO Clinical Practice Guidelines. Ann Oncol 2020;31(12):1650-63.

7. Greenspan SL, Brufsky A, Lembersky BC, Bhattacharya R, Vujevich KT, Perera S, et al. Risedronate prevents bone loss in breast cancer survivors: a 2-year, randomized, double-blind, placebo-controlled clinical trial. J Clin Oncol 2008;26(16):2644-52.

8. Van Poznak C, Hannon RA, Mackey JR, Campone M, Apffelstaedt JP, Clack G, et al. Prevention of aromatase inhibitor-induced bone loss using risedronate: the SABRE trial. J Clin Oncol 2010;28(6):967-75.

9. Lester JE, Dodwell D, Purohit OP, Gutcher SA, Ellis SP, Thorpe R, et al Prevention of anastrozole-induced bone loss with monthly oral ibandronate during adjuvant aromatase inhibitor therapy for breast cancer. Clin Cancer Res 2008;14(19):6336-42.

10. Gnant M, Mlineritsch B, Stoeger H, Luschin-Ebengreuth G, Knauer M, Moik M, et al. Zoledronic acid combined with adjuvant endocrine therapy of tamoxifen versus anastrozol plus ovarian function suppression in premenopausal early breast cancer: final analysis of the Austrian Breast and Colorectal Cancer Study Group Trial 12. Ann Oncol 2015;26(2):313-20.

11. Coleman R, de Boer R, Eidtmann H, Llombart A, Davidson N, Neven P, et al. Zoledronic acid (zoledronate) for postmenopausal women with early breast cancer receiving adjuvant letrozole (ZO-FAST study): final 60-month results. Ann Oncol 2013;24(2):398-405.

12. Brufsky AM, Harker WG, Beck JT, Bosserman L, Vogel C, Seidler C, Jin L, et al. Final 5-year results of ZFAST trial: adjuvant zoledronic acid maintains bone mass in postmenopausal breast cancer patients receiving letrozole. Cancer 2012;118(5):1192-201.

13. Llombart A, Frassoldati A, Paija O, Sleeboom HP, Jerusalem G, Mebis J, et al. Immediate administration of zoledronic acid reduces aromatase inhibitor-associated bone loss in postmenopausal women with early breast cancer: 12-month analysis of the E-ZO-FAST trial. Clin Breast Cancer 2012;12(1):40-8.

14. Gnant M, Pfeiler G, Steger GG, Egle D, Greil R, Fitzal $\mathrm{F}$, et al. Adjuvant denosumab in postmenopausal patients with hormone receptor-positive breast cancer (ABCSG-18): disease-free survival results from a randomised, double-blind, placebo-controlled, phase 3 trial. Lancet Oncol 2019;20(3):339-51.

15. Smith MR, Egerdie B, Hernández Toriz N, Feldman R, 
Tammela TL, Saad F, et al. Denosumab in men receiving androgen-deprivation therapy for prostate cancer. N Engl J Med 2009;361(8):745-55.

16. Yoneda T, Michigami T, Yi B, Williams PJ, Niewolna M, Hiraga T. Actions of bisphosphonate on bone metastasis in animal models of breast carcinoma. Cancer 2000;88(12 Suppl):2979-88.

17. Paterson AH, Anderson SJ, Lembersky BC, Fehrenbacher L, Falkson CI, King KM, et al. Oral clodronate for adjuvant treatment of operable breast cancer (National Surgical Adjuvant Breast and Bowel Project protocol B-34): a multicentre, placebo-controlled, randomised trial. Lancet Oncol 2012;13(7):734-42.

18. Powles T, Paterson A, McCloskey E, Schein P, Scheffler B, Tidy A, et al. Reduction in bone relapse and improved survival with oral clodronate for adjuvant treatment of operable breast cancer [ISRCTN83688026]. Breast Cancer Res 2006;8(2):R13.

19. Coleman R, Cameron D, Dodwell D, Bell R, Wilson C, Rathbone E, et al. Adjuvant zoledronic acid in patients with early breast cancer: final efficacy analysis of the
AZURE (BIG 01/04) randomised open-label phase 3 trial. Lancet Oncol 2014;15(9):997-1006.

20. Early Breast Cancer Trialists' Collaborative Group (EBCTCG). Adjuvant bisphosphonate treatment in early breast cancer: meta-analyses of individual patient data from randomised trials. Lancet 2015;386(10001):1353-61.

21. Gralow JR, Barlow WE, Paterson AHG, M'iao JL, Lew DL, Stopeck AT, et al. Phase III randomized trial of bisphosphonates as adjuvant therapy in breast cancer: S0307. J Natl Cancer Inst 2020;112(7):698-707.

22. Coleman R, Finkelstein DM, Barrios C, Martin M, Iwata $\mathrm{H}$, Hegg R, et al. Adjuvant denosumab in early breast cancer (D-CARE): an international, multicentre, randomised, controlled, phase 3 trial. Lancet Oncol 2020;21(1):60-72.

23. Smith MR, Saad F, Coleman R, Shore N, Fizazi K, Tombal B, et al. Denosumab and bone-metastasisfree survival in men with castration-resistant prostate cancer: results of a phase 3, randomised, placebo-controlled trial. Lancet 2012;379(9810):39-46. 